Voix et Images

volxetimages

\title{
Bibliographie de Jacques Brault
}

\section{Roger Chamberland}

Volume 12, numéro 2 (35), hiver 1987

Jacques Brault

URI : https://id.erudit.org/iderudit/200631ar

DOI : https://doi.org/10.7202/200631ar

Aller au sommaire du numéro

Éditeur(s)

Université du Québec à Montréal

ISSN

0318-9201 (imprimé)

1705-933X (numérique)

Découvrir la revue

Citer ce document

Chamberland, R. (1987). Bibliographie de Jacques Brault. Voix et Images, 12(2),

256-264. https://doi.org/10.7202/200631ar d'utilisation que vous pouvez consulter en ligne.

https://apropos.erudit.org/fr/usagers/politique-dutilisation/ 


\section{Bibliographie de Jacques Brault}

\section{par Roger Chamberland, Université Laval \\ POÉSIE}

Trinôme. Poèmes [ en collaboration avec Richard Pérusse et Claude Mathieu], Montréal, Jean Molinet. 1957. $57 \mathrm{p}$.

Mémoire. Montréal. Librairie Déom. 1965, $82 \mathrm{p}$. [ reproduit en entier, sauf quatre poèmes]. Paris, Éditions Bernard Grasset, 1968, 108 p. [ Le poème "Suite fraternelle" parut d'abord dans Parti pris, novembre 1963, p. 39-46 et a été réédité à Ottawa, Éditions de l'U niversité d’Ottawa, 1969. 39 p. ]. Reproduit dans Poésie I. Saint-Lambert. Éditions du Noroît, 1986, $241 \mathrm{p}$.

La Poésie ce main, Paris, Éditions Bernard Grasset. [1971 ]. 116 p.; Montréal, Parti Pris. 1973. 128 p. (Paroles). Reproduit dans Poésie I, Saint-Lambert. Éditions du Noroit, 1986, 241 p.

Poèmes des quatre côtés, Saint-Lambert, Éditions du Noroît, 1975, 95 p.

Les Hommes de paille, Montréal, Editions du Grainier. 1978. [n.p.]. [Gravures de Marie Anastasie ].

L'En dessous l'admirable. Montréal. Les Presses de l'Université de Montréal, 1975. 51 p. (Lectures). [Traduit sous le titre] Within the M!ster!. Translated and préface by Gertrude Sanderson, Montréal, Guernica, 1986. 50 p.

Migrations, avec des bois gravés de Monique Charbonneau. Saint-Marc. Éd. de la Serfouette, 1979.

Vingt-quatre murmures en novembre avec des eaux-fortes de Janine Leroux-Guillaume, SaintLambert, Éditions du Noroit. [n.p.].

Trois fois passera précédé de Jour et Nuit, Saint-Lambert. Éditions du Noroît. 1981, 87 p [Collages de Célyne Fortin].

Moments fragiles, Saint-Lambert, Editions du Noroît, 1984, 109 p. [ Lavis de l'auteur]. [ Traduit sous le titre] Fragile Moments. Translated by Barry Callaghan. Toronto. Exile, 1985, 93 p.

Ductus (version quadrilingue: français, anglais, italien. allemand) avec une calligraphie et des eaux-fortes de Martin Dufour, Saint-Lambert, Éditions du Noroît, 1984.

La Naissance des nuages, avec des eaux-fortes de Lucie Lambert, Shawinigan. Éd. Lucie Lambert, 1984.

\section{ROMAN}

Agonie, Montréal, Éditions du Sentier, 1984.77 p. [Avec des illustrations de Martin Dufour]: Boréal Express, $1985,77 \mathrm{p}$.

\section{NOUVELLES}

Nouvelles.' [en collaboration avec André Major et André Brochu], Montréal, A.G.E.U.M., 1963, 139 p. (Cahiers, no 6).

\section{ESSAI}

Alain Grandbois, Montréal. Fides, 1958.96 p. (éd. revue et corrigée. 1967).

La Poésie et nous [ en collaboration avec Michel van Schendel. Gilles Hénault. Wilfrid Lemoyne, Yves Préfontaine ], Montréal, l'Hexagone, 1958,93 p.

Alain Grandbois, Montréal, l'Hexagone [ et ] Paris, Seghers, 1968, 183 p. (Coll. Poètes d'aujourd'hui no 172).

Chemin faisant. Essais, Montréal, la Presse, 1975, 150 p. (Échanges). 


\section{EDITION CRITIQUE}

Saint-Denıss Garneau: Oeuvres. Texte établi. annoté et présenté par Jacques Brault et Benoît Lacroix. Montréal. les Presses de l'Université de Montréal, 1971, 1320 p. (Bibliothèque des lettres québécoises).

\section{THÊÂTRE}

Trois partitions, Montréal. Leméac. 1972. 195 p. [ Deux pièces parurent d'abord dans les Ecrits du Canada français, «la Morte-Saison. Théâtre», vol. XXV, 1969. p. 9-20; "Quand nous serons heureux. Théâtre", vol. XXIX, 1970, p. 199-248 ].

\section{ETUDES}

\section{Sur l'ouvre en général}

[ANONYME]. "Arts et spectacles. Jacques Brault remporte le prix France-Canada", le Devoir, vol. LIX. no 212 (23 octobre 1968). p. 12.

- «Wilfrid Lemoyne s'entretient avec Jacques Brault», Ici Radio-Canada/Télévision. vol. Il. no 21 (semaine du 21 au 27 mai 1977), p. 7.

- "Profil. Jacques Brault", Ériture française dans le monde, vol. 2, nos 1-2 (octobre 1980), p. 78 .

ARCHAMBAULT, Gilles, "Jacques Brault: la pléthore des éditeurs", Livre d'ici, novembre 1986. p. 17.

BEAULIEU. Michel. "la Transgression chez Jacques Brault", Presqu'Amérique. vol. I, no 3 (décembre 1971), p. 25-26.

BELLEAU, André, “Quelques remarques sur la poésie de Jacques Brault», Liberté, vol. 12, no 2 (mars-avril 1970), p. 85-92.

BONENFANT, Joseph et Michel Lemaire, "Jacques Brault", Daniel Conty, Alain Rey, JeanPierre de Beaumarchais. Dictionnaire des littératures de langue française. $\mathrm{t}$. I. Paris, Bordas, 1984. p. 322.

BOUVIER, Luc, "l'Analyse des personnages dans la poésie de Jacques Brault». Thèse de maîtrise ès arts, Université Laval, 1982 , vi-89 p.

EMONT, Bernard, "Au royaume d'amour et de mort, situation d'un poète: Jacques Brault ", Livres et Auteurs québécois 1970. Montréal. Éditions Jumonville. 1971, p. 280-292.

GODBOUT. Jacques. "A quoi servent les poètes?», le Maclean. vol. 16, no 8 (août 1976). p. 46-47.

GRANDPRÉ. Pierre de, "Coulée centrale du lyrisme. Trinôme (1957)», Histoire de la littérature française du Québec, t. 111 (1945 à nos jours). La Poésie, Montréal, Librairie Beauchemin Limitée. 1969. p. 179-180.

HAMBLET. Edwin. "la Poésie québécoise», Canadian Literature, no 76 (Spring 1978), p. 94-95.

HAMEL, Réginald. John Hare et Paul Wyczynski, «Jacques Brault», Dictionnaire pratique des écrivains québécois, Montréal, Fides, 1976, p. 85-86.

LEFRANÇOIS, Alexis, "Entretien avec Jacques Brault», Liberté, vol. XVIl, no 4 (juillet-aout 1975), p. 66-72.

LEMAIRE, Michel, "Jacques Brault dạns le matin», Voix et images, vol. 1I, no 2 (décembre 1976). p. 173-194.

- dans En collaboration. The Canadian Encyclopedia, volume 1. A-For, Edmonton. Hurtig Publishers, 1985, p. 214.

LEMAÎTRE, Henri, "Brault, Jacques», Dictionnaire Bordas de littérature française et francophone, Paris. Bordas, 1985, p. 125.

MAILHOT, Laurent et Pierre Nepveu compilateurs, «Jacques Brault" la Poésie quéhécoise des origines à nos jours, Anthologie. Montréal, l'Hexagone, 1986, p. 366-376. (Typo).

MARTEL, Réginald, "Brault: 'J'écris... pour écrire'», la Presse, vol, 86, no 50 (28 février 1970), p. 35 . 
- "Jacques Brault. L'Aventure interminable", la Presse, vol. 102, no 186 (25 octobre 1986). p. E-1, E-9.

MAUGEY, Axel, «Hommage à un poète du Québec: Jacques Brault», l'Action nationale, vol. 59. no 6 (février 1970). p. 592-604.

— «Jacques Brault". Poésie et Société au Québec 1937-1970. Québec. PUL. 1972. p. 209-220.

MELANCCON, Robert, "les Travaux de l'apprenti», le Devoir. vol. LXX. no 134 (9 juin 1979). p. $15-16$.

ROYER, Jean, "Jacques Brault. Du côté du silence», le Devoir, vol. LXX, no I34 (9 juin 1979), p. 15-16. Reproduit dans Érivains contemporains. Entretien 1: 1976-1979. Montréal. l'Hexagone, 1982, p. 233-238.

- «Jacques Brault. Leçons de solitude», le Devoir, vol. LXXVII, no 186 (25 octobre 1986). p. Cl, C7.

SOTIROPOULO-PAPALEONIDAS, Irêne. Jacques Brault. Théories/pratique de la traduction. Sherbrooke, Éditions Didon, 1981, 112 p.

Union des écrivains québécois et [ Légaré, Yves], "Jacques Brault", Dictionnaire des écrivains québécois contemporains, Montréal, Québec/Amérique, 1983. p. 76-77.

\section{Trinôme}

[ANONYME], "Trinôme, un recueil signé par trois poètes ", la Presse, vol. LXXIII, no 198 (8 juin 1957), p. 65.

BERTHIAUME, Pierre. "Trinôme", Dictionnaire des auvres littéraires du Québec, t. III. 1940-1959. Montréal. Fides, 1982. p. $1016-1018$.

CHAPUT-ROLLAND. Solange, "les Livres. Trinôme", Points de vue, vol. 2, no 12 (septembre 1957), p. 32.

GARCIA, Juan, "la Poésie de Jacques Brault», Liberıé. vol. VII, no 6 (novembre-décembre 1965), p. 563-566.

GOULET. Elie, "les Livres canadiens. Pérusse, Brault, Mathieu, Trinôme», Culture, vol. XVIll, no 4 (décembre 1957), p. 451.

GRANDPRE. Pierre de. a la Vie des lettres. Notre jeune poésie du chiffre noir. Boréal-Trinôme - Tante Élise", le Devoir, vol. XLV1I1, no 157 (6 juillet 1957), p. II.

LEMOYNE, Wilfrid, «Livres. Trinôme», Vie des arts, no 7 (été 1957), p. 31.

PILON, Jean-Guy, "le Sens des faits. Les cloîtres de l'été", Revue dominicaine, vol. 61, (avril 1955), p. 180-182.

PREFONTAINE, Yves. «Poésie pas morte», Maintenant, no 42 (Juin 1965), p. $211-213$.

ROBERT, Guy, "l'Esprit des livres. Trinôme», Revue dominicaine, vol. 63 (novembre 1957). p. 252.

\section{Nouvelles}

ARCHAMBAULT, Gilles, "Nouvelles de André Major, Jacques Brault et André Brochu», Livres et Auteurs canadiens 1963, Montréal, Éditions Jumonville, 1964, p. 39-40.

GAUVIN, Lise, "Nouvelles", Dictionnaire des ceuvres hittéraires du Québec, tome IV, 1960-1969. Montréal. Fides, 1984, p. 624-625.

\section{Mémoire}

[ANONYME], "la Vie littéraire. Carnet", le Droit, vol. 57, no 202 (22 novembre 1969), p. 14. — "Prix France-Canada à Jacques Brault", Québec Amérique, (avril 1969), p. 36.

AUDEJEAN. Christian, "Mémoire de Jacques Brault", Esprit, novembre 1968, p. 596.

BARBERIS, Robert, "Aux conférences J. A. de Sève. Miron le Magnifique [ Mémoire]", le Quartier latin. vol. XLVIII, no 32 (17 février 1966). le Cahier des arts et des lettres, vol. II, no 16. p. 1, 3 .

- "Mémoire de Jacques Brault», Maintenant, no 81 (15 novembre - 15 décembre 1968), p. $279-280$. 
BEAUlIEU. Michel, "Faisceaux de la poésie québécoise" Presqu'Amérique, vol. 1. no 2 (novembre-décembre 1971), p. 22-24.

- "La Transgression chez Jacques Brault". Presqu'Amérique, vol. I, no 3 (décembre 1971), p. 25-26.

BELLEAU, André, "Quelques remarques sur la poésie de Jacques Brault", Liberté, vol. XIl, no 2 (mars-avril 1970), p. 85-93.

BOSQUET, Alain, “Un succès Jacques Brault à Paris», le Devoir, vol. LIX, no 27 (juillet 1968). p. 7 [ reproduit du Monde].

BOSQUET. Alain. «Jacques Brault». le Monde. 20 juillet 1968.

CHAPPUIS. Pierre. "Jacques Brault". Gazette de Lausanne, septembre 1968.

CLUNY, Claude Michel, "Jacques Brault: Mémoire et Alain Grandboism, le Devoir, vol. 85. no 103 (3 mai 1969), p. 18 [ reproduit de la $N R F$. février 1969].

COUTURE, André, «la Vie littéraire. La mort, cette éternelle vie! » le Droit, vol. 57, no 207 (29 novembre 1969). p. 17.

EMONT. Bernard, "Au royaume d'amour et de mort. situation d'un poète: Jacques Brault". Lives e't Auteurs québécois 1970, p. 280-292.

GARCIA. Juan, "la Poésie de Jacques Brault", Liberté, vol. VII, no 6 (novembre-décembre 1965). p. 564-566.

GAULIN. André, "Mémoire". Dictionnaire des auvres littéraires du Québec, tome IIl, 19601969. Montréal, Fides, 1984, p. 552-556.

GAY. Paul, «la Vie littéraire. La jeune poésie». le Droit, vol. 56, no 85 (6 juillet 1968), p. 7.

- “la Vie littéraire. Suite fraternelle», le Droit, vol. 57, no 195 (15 novembre 1969), p. 12.

GODEL. Vahé, "Un sens aigu de l'épopée quotidienne», la Tribune dé Genève. 29 mại 1968.

GODIN, Gérald, «Un paỳs adoré et honni», le Magazine Maclean, vol. 8, no 7 (juillet 1968). p. 47.

LABONTÉ. René. "le Québec entre trois océans. Ambiguïté du référent au mot pays dans un poème de Jean-Guy Pilon et Jacques Braultm, l'Action nationale. vol. 67. no 7 (mars 1978), p. 566-575.

LACÔTE, René, "Jacques Brault", les Lettres françaises, 15-21 avril 1965.

LOSSIER, Jean-Georges, «Un peuple ivre de vents et de femmes». Journal de Genève, 4 janvier 1969.

MAILHOT, Laurent. "Contre le temps et la mort: Mémoire de Jacques Brault". Voix el Images du pays, vol. 111 (1970), p. 124-144 [ reproduit dans Ellipse, no 7. (printemps 1971). p. 30-50].

MAJOR. André, “Mémoire de Jacques Brault», Livres et Auteurs canadiens, 1965. 'Montréal, Éditions Jumonville. 1966, p. 97-98.

- “Pour une pensée québécoise", Cahiers de Sainte-Marie, no 4 (avril 1967), p. 125-131.

- "Jacques Brault: "Ici on est acculé à l'invention, c'est une chance extraordinaire"", le Devoir, vol. 83. no 252 (31 octobre 1967), p. viii.

- "la Semaine littéraire. Mémoire», le Devoir, vol.L.IX. no 117 (18 mai 1968), p. is.

- "la Vie littéraire. De Jacques Brault: Mémoiren. le Droit, vol. 52, no 73 (27 mars 1965), p. 7.

- «Livres en français. Poésien. University of Toronto Quarterly. vol. XXXVIII, no 4 (July 1969), p. 478-486 [v. p. 478-480]:

MARCHAND, Olivier, “Littérature. Noblesse séculaire de la poésie», la Presse, vol. LXXXIV, no 122 (25 mai 1968), p. 29.

MARCOTTE, Gilles, “Littérature. Poésie des deux rives. Commune présence de René Char. Suite fraternelle de Jacques Brault", la Presse (supp. Arts et Lettres), vol. LXXXI. no 94 (24 avril 1965), p. 4.

- le Temps des poètes, Montréal. HMH. 1969. p. 191-194.

MARTEL, Réginald, «Simples Valeurs de l'utopie» la Presse, vol. LXXXVI, no 62 (20 mars 1971), p. 27. 
MAUGEY. Axel, "Hommage à un poète du Québec: Jacques Brault". l'Action nationale, vol. 59, no 6 (février 1970). p. 592-604.

MELANÇON, André, "Brault (Jacques). Mémoire [...]". Leçures, vol. XI. no 9 (mai 1965), p. 246.

MORA, Edith, "la Muse et le Cosmos», les Nouvelles littéraires, 16 mai 1968.

PARADIS, Suzanne. "Poésie québécoise Jacques Brault. Le poète est d'abord un citoyen scandalisé", le Soleil, vol. 74, no 232 (2 octobre 1971), p. 47.

PELOSSE, Cécile, "la Recherche du pays dans la poésie québécoise de 1945 à 1970 ». Thèse de doctorat ès lettres, Montréal, Université de Montréal, 1974, vi, $473 \mathrm{f}$.

POULIN. Gabrielle. «Mémoire de Jacques Brault», Relations, no 334 (janvier 1969). p. 13-16.

PRÉFONTAINE. Yves, "Poćsie pas morte!", Maintenant, no 42 (juin 1965), p. 211-213 [v. p. 213].

SYLVESTRE. Guy, "la Poésie. Jacqués Brault, M. Laberge, Marie-Anastasie", le Devoir, vol. LVI, no 143 (19 juin 1965), p. 11 [ reproduit dans Québec 65. vol. 2, octobre 1965. p. 90-92 ].

- "Livres en français. La poésie", Universit!' of Toronto Quarterly; vol. XXXV. no 4 (July 1966). p. 503-555 [v. p. 505-506].

THÉBERGE. Jean-Yves. "Un peu de réalité, un peu de rêve. Mémoire», le Canada français, vol. CV, no 45 ( (1er avril 1965). p. 30.

VAN SCHENDEL, Michel, “Poésie québécoise 1960-1965: l'apprivoisement du vertige ou la rencontre des nouvelles traditions", Livres et Auteurs canadiens 1965. Montréal, Éditions Jumonville. 1966. p. 13-22 [v. p. 22].

\section{Alain Grandbois}

«Rencontre avec Jacques Brault: “Grandbois est le maître d'une génération"», le Devoir, vol. 59. no 93 (20 avril 1968), p. 18.

ETHIER-BLAIS, Jean, "Alain Grandbois", Québer. $5^{\varepsilon}$ année (octobre 1968), p. 104-106.

F., S.. "Alain Grandbois", Culture, vol. 21, no 2 (juin 1960), p. 216.

GALLAYS. François, “ Alain Grandbois». Livres et Auteurs canadiens 1968. Montréal, Éditions Jumonville. 1969, p. 128-129.

MAILHOT, Laurent. "Alain Grandbois", Eludes fransaises, vol. 4, no 4. (novembre 1968). p. $\mathbf{4 4 0 - 4 4 2 .}$

RIOUX. Gilles, "Alain Grandbois", Sept-Jours, $2^{\mathfrak{c}}$ année, no 50 (31 août 1968), p. 38-39.

\section{La Poésie ce matin}

[ ANONYME], “Ainsi laisser parler", la Presse, vol. 87, no 246 (23 octobre 1971), p. C-3.

- «Jacques Brault publie une deuxième fois à Paris", Québec-Presse (supp. le Livre), vol. IV, no 13 (26 mars 1972), p. 12.

BEAULIEU, Michel, "la Poésie ce matin», Point de mire, vol. 3, no 6 (20 novembre 1971), p. 40.

BOSQUET, Alain, "Poètes de la parole», Journal de Bruxelles, septembre 1971.

GAY. Paul, «l'Amour et la Mort", le Droit, vol. 60, no 129 (9 septembre 1972). p. 7.

HOULE, Benoît, “Des recueils de deux poètes québécois paraissent à Paris», la Presse, vol. 88. no 34 (20 mars 1972), p. C-8.

— «la Poésie québécoise à l'assaut de Paris», le Soleil, vol. 76, no 72 (21 mars 1972), p. 24 [ reproduit dans le Droit, vol. 59. no 305 (25 mars 1972). p. 20].

KEARNS SANDERSON, Gertrude, "La Poésie ce matin", the Antigonish Review. vol. 3, no 8 (hiver 1972): p. 100-102.

LEMAIRE. Michel. "Jacques Brault dans le matin", Voix et images, vol. 2, no 2 (décembre 1976), p. 173-194.

MAJOR, Jean-Louis, “Livres en français. La poésie". University of Toronto Quarterly, vol. 67. no 4 (été 1972). p. 348.

MARCOTTE. Gilles, «Chroniques. La poésie - pour l'âme», Érudes françaises, vol. VIII, no I (février 1972), p. 87-100. 
MAURIAC. Claude. "les Écrivains du mardi", le Figaro, 12 septembre 1972.

ROUSSELOT. Jean. "Un peu de parole....m, les Nouvelles liméraires, 10 septembre 1971.

THÉBERGE, Jean-Yves, "Jacques Brault chez Parti pris", le Canada français vol. CXIV, no II (8 août 1973), p. 72.

WYCZYNSKI, Paul, "la Poésie ce matin de Jacques Brault". Livres et Auteurs québécois 1971 , Montréal, Éditions Jumonville, 1972, p. 148-151.

\section{Trois partitions}

[ ANONYME], " "Quand nous serons heureux"», Ici Radio-Canada, vol. 3, no 40 (27 septembre 1969). p. 4.

— "Quand nous serons heureux". le Livre canadien. vol. 1, no 199, 1970.

- "Trois partitions". le Livre canadien. vol. 3. no I2I. 1972.

CÔTÉ. Fernand, "Deux textes de Jacques Brault, en première partie des "Beaux Dimanches" suivis d'un documentaire, "Victoire sur l'Annapurna" ", Ici Radio-Canada, vol. V, no 25 (du 12 au 18 juin 1971), p. 5.

GOBIN. Pierre. le Fou et ses doubles. Figures de la dramalurgie quéhécoise. Montréal, les Presses de l'Université de Montréal, 1978, p. 28, 46, 48-51, 110, 126-131.

HÉBERT, François, "Notre conscience mythique (Théâtre de Jacques Brault)", Livres et Aluleurs québécois, 1972. Montréal, Éditions Jumonville, 1973, p. 118-120.

MAlLHOT. Laurent, "Chroniques. Le Théâtre - des missionnaires aux sauvages ou du sacré au sacrant ". Etudes françaises, vol. VIII, no 4 (novembre 1972), p. 409-427 [ v. p. 419-423].

- "Letters in Canada [...] Théâtre", University of Toronto Quarterly, vol. XLIl, no 4 (Summer 1973), p. 363-366 [v. p. 364].

— "Trois partitions". Dictionnaire des aeuvres littéraires du Québer, t. 111. 1940-1959. Montréal. Fides, 1982, p. 891-892.

MAILLET, Antonine, postface du recueil Trois partitions, p. 187-193.

PAGÉ, Pierre et Renée LEGRIS. Répertoire des dramatiques québécoises à la télévision. $1952-$ 1977, Montréal. Fides, 1977, p 50.

PONTAUT, Alain. préface du recueil, p. 9-19.

- Distionnaire critique du théâtre québécois, Montréal, Leméac, 1972. p. 24-25.

RINFRET. Edouard-G.. le Théâtre canadien d'expression francaise. Répertoire analvtique des origines à nos jours. [ préface de Robert Choquette]. Montréal, Leméac. 4 vol. (v. 1 t. I: [ 1975], p. 88-89; t. IV: Télévision (1952-1973) et Index. [ 1978], p. 21-22.

SAINT-ONGE. Paule, "Trois partitions", Châtelaine, vol. 13, no 9 (septembre 1972), p. 16.

\section{Saint-Denys Garneau: Oeuvres}

[ANONYME], "Saint-Deny's Garneau: Oeuvres", le Livre canadien, vol. 2. no 4, 1971.

TOUSSAINT, Fernand, "Saint-Denys Garneau: Oeuvres», Action pédagogique, nos 19-20 (1971), p. 92.

\section{Poèmes des quatre côtés}

ALYN, Marc, "Jacques Brault», le Figaro. 21 juin 1975.

[ANONYME], "Poèmes des quatre côtés», le Livre canadien. vol. 7, no 213 (juin 1976).

AMPRIMOZ. Alexandre, [ Poèmes des quatre côtés]. Tamarack Review', no 70 (Winter 1977), p. $72-80$, p. $75-77$.

AUDET, Nö̈l, "Poèmes des quatre côtés de Jacques Brault", Voix et images, vọl. I, no I (septembre 1975), p. 131-134.

BEAUSOLEIL. Claude, "Poèmes des quatre côtés", Hobo / Québec, no 25 (septembredécembre 1975), p. 11.

BOURQUE, Paul-André. "Jacques Brault. Poèmes des quatre côtés. Éditions du Noroît". Livres et Auteurs québécois 1975. Québec. les Presses de l'Université Laval, 1976, p. 110-112.

COULOM BE, Gilbert, "Brault: Poèmes des quatre côtés de la traduction non traduite [...]", le Canada français, vol. CXV, no 50 (7 mai 1975), p. 48. 
DIONNE. René. "Livres en français. La Poésie. [ Poèmes des quatre côtés]", University of Toronto Quarterly, vol. 45, no 4 (Summer 1976), p. 349.

GAULIN. André, "Nouvéautés. Poésie. Poèmes des quarre côtés". Québec frantrais, no 19 (octobre 1975), p. 7.

GODBOUT, Jacques, «les Livres. Au nom du peuple», le Maclean, vol. XV, no 8 (août 1975), p. 8.

HAECK, Philippe, "la Poésie. Un travail de mélancolique, signé Jacques Brault". Ie Dev'oir. vol. LXVII. no 101. 3 mai 1975, p. 17.

LEFRANÇOIS, Alexis, "Accueillir le plus profond rêve du temps", Liherré, vol. XVII, no 4 (juillet-août 1975). p. 57-65.

PAGÉ, Raymond, "Quebec Literature 1914-1975. Part 1: Poetry and Novels». Chelsea Journal. vol. II, no 2 (March / April 1976), p. 79-81.

POISSON, Roch, "Poèmes des quatre côtés», Nous, vol. 3, no 1 (juin 1975), p. 17.

PONTAUT, Alain, "En toutes lettres. Quand traduire est le fait d'un poète", le Jour, vol. II. no 30 (7 avril 1975). p. 13.

— «Pòmes des quatre côtés», Vient de paraitre, vol. 11. no 4 (novembre 1975. p. 42.

JACQUES, Réjean. «Lapointe. Brault et les mythes», la Presse, vol. 91 , no 105 (3 mai 1975), p. E-4-11.

RICARD. François, «Littérature québécoise. Livres de poésie (Jacques Brault. Paul Chamberland)m, Liberté, vol. XVII, no 4 (juillet-août 1975), p. 100-1II [v. p. 100-106].

ROYER, Jean, «Jacques Brault: le poème sur le chemin de l'écriture", le Soleil, vol. 79, no 154 (28 juin 1975). p. D-9.

\section{L'En dessous l'admirable}

[ ANONYME], "l'En dessous l'admirable», le Livre canadien, vol. 7, no 173 (mai 1976).

BOUVIER. Luc, "l'En dessous l'admirable», Livres et Auteurs québécois 1976. p. 135-138.

DIONNE, René, "Letter in Canada. Poésie", University of Toronto Quarter!!', vol. 45, no 4 (summer 1976). p. 350-351.

DOSTIE, Gaétan. "Livres. Brault et Guay ou les alliances simples». Le Jour, vol. II, no 298 (27 février 1978). p. 10.

GAUl.IN. André, "Nouveautés. Poésie. L'En dessous ladimirable», Quéhec français, no 21 (mars 1976), p. 10.

GODBOUT, Jacques; "À quoi servent les poètes?", le Maclean, vol. 16, no 8 (août 1976), p. 46-47.

HAECK, Philippe, “Du côté de l'idéalisme». Chroniques, no 15 (mars 1976). p. 41-42.

HAMBLET, Edwin. "la Poésie québécoise». Canadian Literature, no 76 (Spring 1978), p. 94-95.

HEBERT, François, "Livres. Des arbres dans le bois: trois poètes", le Jour, vol. II, no 275 (30 janvier 1976). p. 16.

MAUGEY, Axel, "Jacques Brault au coeur de l'ombre», Vie des arts, vol. 21, no 84 (automne 1976), p. 57.

PIAZZA. François, “l'En dessous admirable", Montréat-Matin, vol. XLVI, no 197 (18 janvier 1976), p. 9.

POULIN, Gabrielle, «Entre les lignes. L'En dessous l'admirable, de Jacques Brault. Par delà ce pays de trop bref étén, le Droit, vol. 64, no 77 (26 juin 1976), p. 19.

- «Des mémoires d'outre-neige. L'En dessous l'admirable de Jacques Brault", Relations, vol. 36, no 417 (juillet-août 1976). p. 221-222.

RICARD, François, "Livres de poésie (Jacques Brault. Paul Chamberland)», Liberté, no 100. vol. 17, no 4 (juillet-août 1975), p. 110-111.

- "Jacques Brault, la reconstitution d'un voyage de l'en dessous à l'admirable", Hebdos-Québec, mars 1976. 
ROYER, Jean. "Jacques Brault: l'En dessous l'admirable", le Soleil. vol. 80, no 30 (31 janvier 1976), p. D-6.

VACHON, Georges-André, "Jacques Brault à la recherche d'un lieu commun", Éludes françaises, vol. 13, nos I-2 (avril 1977), p. 18I-188.

WEISS, JONATHAN-M., "Jacques Brault. L'En dessous l'admirable", French Revien', February 1979. p. 508-509.

\section{Chemin faisant}

[ ANONYME], “Ouvrages reçus», le Canada français, vol. CXVI, no 34 (21 janvier 1976), p. 47.

- "Chemin faisan/". le Livre canadien, vol. 7, no 172. (mai 1976).

BEAUSOLEIL. Claude. "Lire aujourd'hui. Trois essais. Trois rapaillages différents [ Chemin faisant ]". Hobo / Québec, nos 29-30 (mai-août 1976), p. 5.

BOUCHER, Jean-Pierre. «Jacques Brault Chemin faisant". le Devoir, vol. LXVIll, no 13 (17 janvier 1976), p. 15.

BOURBONNAIS, Nicole, "les Études littéraires. Chemin faisan de Jacques Brault", L.ettres quéhécoises. no 2 (mai 1976), p. 27-28.

GAULIN, André, "Nouveautés. Essais. Chemin faisant”, Québec fraņ̧ais, no 22 (mai 1976), p. 8.

HÉBERT, François, “Chemin faisant", Livres et Auteurs québécois 1976, Québec. les Presses de I'Université Laval, 1977, p. 209-210.

MARTEL. Réginald, "Quand le silence dira la parole», la Presse, vol. 91, no 308 (27 décembre 1975). p. C-2.

ROYER, Jean. "De la vacuité de la littérature à l'importance de l'écriture», le Soleil, vol. 80. no 24 (24 janvier 1976). p. D4.

\section{Trois fois passera}

BAYARD, Caroline, «Livres en français. Poésie», University of Toronto Quarterly, vol. 51, no 4 (Summer 1982). p. 357-360.

BEAUSOLEIL. Claude, «Jacques Brault. Une intimité sans concession», le Devoir, vol. LXXII. no 140 (22 août 1981), p. 15.

BELLEFEUILLE, Normand de. "Trois fois passera», Spirale, no 21 (septembre 1981), p. 4-5.

BROCHU, André, «Rétrospectives et prospectives», Vbix et images, vol. 7, no 3 (printemps 1982), p. 586-587.

GIROUX, Robert, "Trois fois passera précédé de Jour et Nuit", Livres et Auteurs québécois 1981, Québec. PUL. 1982, p. 86-87.

HAECK. Philippe. "Trois fois passera, précédé de Jour et Nuit", Estuaire, no 23 (printemps 1982). p. 116-118.

HÉBERT, François, “Trois fois passera". Liberté, vol. 23, no 6 (novembre-décembre 1981), p. $112-113$

LEMAIRE, Michel, «Jacques Brault. L'Orfèvre et l'Artisan", Lettres quéhécoises, no 23 (automne (981), p. 64-65.

\section{Agonie}

BOIVIN. Jean-Roch, "Jacques Brault ou l'éloquence de la retenue", Montréal ce mois-cí, septembre 1985, p. 22

CLOUTIER. Guy, "l'Homme navré», Nuit Blanche, décembre 1985, p. 4.

COSSETTE. Gilles, “Récit», Lettres québécoises, no 36 (hiver 1984-1985). p. 2426.

GUENETTE. Daniel, "Agonie», Nos livres, février 1985. p. 28-30.

HÉBERT, François, "Un poète philosophe et un philosophe poète", le Devoir, vol. LXXVI, no 280 ( $1^{\text {er }}$ décembre 1984). p. 23.

MARCOTTE. Gilles. "Le Champion toutes catégories", l'Actualité, vol. 40, no 10 (octobre 1985). p. 147. 
MARTEL, Réginald, "Mourir comme des oiseaux", la Presse, 10 août 1985, p. B3. MICHAUD, Ginette, "l'Épreuve de la lecture", Spirale, no 48 (décembre 1984). p. 9.

\section{Moments fragiles}

BELANGER, Daniel, “l'Espace-temps nuitamment recherché: Moments fragiles". Leurres québécoises, no 34 (été 1984), p. 34-35.

BROCHU, André, «Ecriture en locomobile", Voix ef images, vol. 10, no I (automne 1984), p. $151-162$.

GIGUËRE. Richard, "YOmbre de Saint-Denys Garneau [... ]". Lettres québécoises, no 35 (automne 1984-. p. 40-43.

MARCHAMPS, Guy. «Moments fragiles", le Sahord, Trois-Rivières, automne 1984, p. 14. 